\title{
Ellipsis in Finnish Sign Language
}

\section{Tommi Jantunen}

This paper deals with syntactic ellipsis in clauses in Finnish Sign Language (FinSL). The point of departure for the paper is the observation, confirmed by several studies, that clauses in FinSL are often syntactically incomplete. Building on this, the paper first describes how all core-internal clausal material may be elided in FinSL: core arguments in clauses with a verbal nucleus, core-internal NPs in clauses with a nominal nucleus, and even nuclei themselves. The paper then discusses several grammatical contexts which especially favor ellipsis in FinSL. These are question-answer pairs, two-clause coordinated structures, topic-comment structures, blend structures, and structures containing gesturally indicating Type 2 verbals. Finally, the paper argues that FinSL conforms to the main characteristics of a discourse-oriented language, and that FinSL clauses are not highly governed units syntactically.

Keywords clause, discourse-orientation, ellipsis, Finnish Sign Language, sign language, syntax

Sign Language Centre/Department of Languages, P. O. Box 35 (F), FI-40014

University of Jyväskylä, Finland. tommi.j.jantunen@jyu.fi

\section{INTRODUCTION}

This paper discusses and analyzes SYNTACTIC ELLIPSIS in clauses in Finnish Sign Language (FinSL). Syntactic ellipsis is the most recognized type of ellipsis in the literature (e.g., Huang 2000, Engberg-Pedersen 2002, McShane 2005, Johnston \& Schembri 2007). It is a phenomenon involving the optional non-expression of a lexeme or a phrase in a syntactic configuration (e.g., The man stepped in and $\emptyset$ saw the woman in which the word man is elided in the second clause, indicated with Ø) (McShane 2005). Another major type of ellipsis is SEMANTIC ELLIPSIS, in which semantically significant elements are left unexpressed without any syntactic gap (e.g., in the passive sentence The game was participated in from all over the country, the semantic agent is not expressed, yet there is no syntactic gap) (McShane 2005). In this paper, semantic ellipsis and consequent phenomena, such as the deliberate defocusing of the agent (i.e., passive; Shibatani 1985), are excluded from the discussion.

The present work on ellipsis is part of a larger project investigating the unit clause in FinSL. More specifically, the discussion of ellipsis is motivated by several studies which have indicated that clauses in FinSL and also in other sign languages are frequently realized in a way that could be considered to be incomplete. With FinSL, Jantunen (2008) was the first to document the fact that the semantic argument 
structure of transitive FinSL verbals may be left syntactically unexpressed. He noted that this type of elision of core arguments is relatively common in FinSL but he did not provide any numbers for the possible frequency of the phenomenon. The issue was later taken up by Ala-Sippola (2012). In her study, Ala-Sippola investigated the expression of the semantic role of agent in transitive clauses and found that, out of the total of 105 clauses in her data, the agent was left lexically unexpressed in 52 cases (50\%). The frequency of omissions in Ala-Sippola's data was considerable, yet it agrees fully with the numbers presented for other sign languages. For example, in their large-scale quantitative study on pro drop, Wulf et al. (2002) found that American Sign Language expressed only $35 \%$ of its subject-like core arguments belonging to the class of pronouns (they also showed that the presence of the pronouns correlated with several sociolinguistic factors, including, for example, English influence: subjectlike pronouns were expressed significantly more in linguistic contexts which were influenced by English-derived structures such as fingerspelled copular structures or loan translations). Similar observations have also been made regarding Danish Sign Language (Engberg-Pedersen 2002) and Australian Sign Language (Johnston \& Schembri 2007).

The paper builds on the observation that ellipsis is very frequent in FinSL. The phenomenon is approached here from three different perspectives. First, because not much is known about the possible targets of ellipsis in FinSL clauses, the paper analyzes and describes what core-internal clausal elements (e.g., core arguments, nuclei) may be elided in FinSL. According to McShane's (2005) typological theory of ellipsis, this also corresponds to answering the first major question concerning the occurrence of ellipsis in any language. Second, on the basis of data and findings from other studies, native intuitions, and typological knowledge about ellipsis, the paper identifies and analyzes some major syntactic configurations and other factors that especially encourage ellipsis in FinSL. Because this work catalogues the grammatically governed circumstances in which certain elements of the clause may remain unexpressed, it is assumed that it will be of great use in future empiricallybased analysis of FinSL clauses; this sort of work is considered to be of primary importance also in McShane's theory. Finally, the paper discusses some of the consequences that the high frequency of ellipsis has on the analysis of FinSL and on its clauses. On the one hand, this discussion centers around the question of the status of FinSL as a discourse-oriented language; on the other, it focuses on the status of the clause in the description of FinSL syntax.

The theoretical framework of the present work is Basic Linguistic Theory (Dryer 2001, 2006; Dixon 2010). As noted by Dryer (2001), Basic Linguistic Theory is an informal (as opposed to formal) descriptive framework widely used by linguists to describe and compare grammars of individual languages. As the emphasis of Basic Linguistic Theory is on description, it is often considered to be a framework suitable for the study of undocumented linguistic phenomena, such as the ellipsis in FinSL. In 
general, Basic Linguistic Theory also accords well with the overall FinSL research tradition (see Jantunen 2008, 2009), which has been primarily descriptive.

Methodologically, research into ellipsis cannot be based on any single confined set of data or, for example, on the simple inductive observation method (McShane 2005). Instead, in order to gain knowledge about what may be elided and what not, and in what circumstances, a maximally wide collection of different types of data and methodological means, including grammaticality judgments from native language users, have to be used. In the present work, this is exactly what has been done: the present work builds on the investigation of both isolated clauses and discourse clauses, uses both monologue and dialogue data, and exploits equally native intuitions and existing syntactically analyzed material. All the examples and analyses in the paper have been discussed with native signers. Whenever an example lacks a citation, it is derived directly from the intuitions of native FinSL signers, consulted in informal interviews.

The text is structured as follows. The next two sections first provide the necessary background for the notions of ellipsis (Section 2) and FinSL clauses (Section 3). The following sections then discuss the three main issues in the present work: what core elements may be left out from FinSL clauses (Section 4), what grammatical contexts especially favor ellipsis in FinSL (Section 5), and what consequences the high frequency of ellipsis has on FinSL and its clauses (Section 6). Then follows the conclusion.

\section{ON ELLIPSIS}

According to McShane (2005), ellipsis is a universal property of natural language, but its scope and means of realization may vary considerably in different languages. For example, whereas in English the elliptical phenomenon of ZERO ANAPHORA the reference to the previously mentioned referent by means of zero - is restricted in coordinated complex sentences by an S/A pivot constraint ${ }^{1}$ (i.e., it is possible to say The man came in and saw the woman but not The man came in and the woman saw; Dixon 1994), in Dyirbal the phenomenon is restricted by an S/P pivot (i.e., the latter type of sentence mentioned above is possible, but not the first type; Dixon 1994). On the other hand, in Mandarin Chinese, zero anaphora in coordinated clauses is not conditioned by either of the two constraints (i.e., it is possible to use both of the above types of sentences), the primary recovery method of the unexpressed argument being, eventually, world knowledge (see Huang 2000).

Ellipsis is considered to be a very frequent phenomenon not only in sign languages (Engberg-Pedersen 2002, Wulf et al. 2002, Johnston \& Schembri 2007, Jantunen 2008, Ala-Sippola 2012) but also in spoken languages (McShane 2005), although studies that have explicitly investigated the frequency of ellipsis in spoken language are hard to find. However, one example of such a study is that by Alcántara 
\& Bertomeu (2005). In their work, the researchers analyzed a 50,000-word corpus of spoken Spanish and found that, of a total of 6922 sentence-coded events in the corpus, $522(7.5 \%)$ were elliptical in terms of some part of the sentence. The number is considered to be very high (Alcántara \& Bertomeu 2005) but, interestingly, it is nowhere near the figures presented for sign languages and FinSL. The difference may be explained by the fact that, typologically, Spanish is not classified as a highly discourse-oriented language (McShane 2005) (whereas sign languages in general and FinSL in particular most likely are such languages, see Section 6).

As noted in Section 1, the two main types of ellipsis are syntactic ellipsis and semantic ellipsis (McShane 2005). Of these, the first refers to the omission of a syntactic element while the latter refers to cases in which a semantic element is missing without any syntactic gap. McShane (2005) demonstrates the two types with the examples (1a) and (1b), respectively.

(1) a. Mary got an A on the math test and Louis $\varnothing$ a B.

b. He is reading Tolstoy.

In (1a), the verb got is missing from the second clause and the clause is therefore interpreted as being syntactically elliptical (the specific type of ellipsis is known as gapping, see Sections 4 and 5 below). In (1b), however, there are no syntactic elements missing from the clause. Instead, the clause is interpreted as being semantically elliptical because it actually means that the protagonist is reading 'a book written by Tolstoy', not Tolstoy per se (according to McShane 2005, the existence of semantic ellipsis in this example is proved by the fact that there are languages that, in a similar context, do not allow such semantic omission). Semantic ellipsis is not the focus of the present paper but it is frequently employed, for example, in passive constructions as a form of agent defocusing (Shibatani 1985, McShane 2005).

Syntactic ellipsis may be further divided into two main types: with textual coreference, and without textual co-reference (McShane 2005). Syntactic ellipsis with co-reference means basically that the elided category has an accessible syntactic antecedent; the phenomenon of zero anaphora, among others, is of this type, as is the verb-eliding phenomenon in (1a). In syntactic ellipsis without co-reference, the antecedent is not accessible syntactically; this type is identified with elided elements that have, for example, generic human or non-human referents. The present paper acknowledges the distinction between the two main types of syntactic ellipsis but does not discuss it explicitly.

According to McShane (2005), there are two conditions that must be met for syntactic ellipsis to be possible. The first is that the language must permit ellipsis in the particular construction. Cross-linguistically, the most typical factors that permit ellipsis are a particular type of lexical category (e.g., auxiliaries permit a variety of elliptical phenomena in some languages, including English, so that it is possible 
to say If you are going to buy that, I will $\emptyset$, too; also question words are typical examples of ellipsis-permitting lexemes) and syntactic parallelism (especially twoclause coordinated structures). The second condition is that the content of the elided category must be recoverable. In practice, referents for syntactically elided categories may be recovered on the basis of either the linguistic or extralinguistic context. The first strategy is used, for example, in the construction of (1a). The second strategy, in turn, is at work in the interpretation of the question Should we?, uttered while standing next to a poster advertising a vacation in the Alps: the target of the inquiry is very likely an event 'go on a ski trip'.

Concerning the reasons why ellipsis exists, the most acknowledged one has always been claimed to be the speaker's supposed wish to reduce his or her efforts. However, recent work on ellipsis has also revealed other plausible explanations and functions for ellipsis. For example, Hendriks \& Spenader (2005) have suggested that the elided forms may often contribute different meanings than those of their fully expressed counterparts which may even violate syntactic or semantic constraints; ellipsis often restricts possible interpretations, and allows people to say things that may otherwise be even inexpressible. Moreover, by forcing parts of the discourse to be interpreted together (e.g., saying John walked and talked instead of John walked and John talked, in which there is the referentially ambiguous second occurrence of John; Hendriks \& Spenander 2005), ellipsis is also known to establish discourse coherence (e.g., Halliday 1994, Johnston \& Schembri 2007). When appropriately used, ellipsis may even be recognized as a positive politeness strategy. This function results from the fact that the omission of the part of a message creates a relatively close relationship with the addressee by assuming shared background information (Hendriks \& Spenander 2005).

\section{THE CLAUSE IN FINSL}

This section provides background about the structure of full, non-elliptical clauses in FinSL. Ellipsis in FinSL will be discussed against this background in Sections 4 and 5 .

In FinSL, the investigation of the CLAUSE has been motivated strongly by the typologically-oriented Role and Reference Grammar (RRG) (Van Valin \& LaPolla 1997, Van Valin 2005). In RRG, the structure of the clause is considered to be based on two semantic contrasts (see also, e.g., Dixon \& Aikhenvald 2000, Givòn 2001). The first is that between predicating and non-predicating elements; languages tend to distinguish predicating elements from those not predicating (Van Valin 2005). The second is the contrast between the arguments of the predicate and non-arguments; languages tend to make the distinction between units required by the semantics of the predicate and those not required by it (Van Valin 2005). The predicate, which 


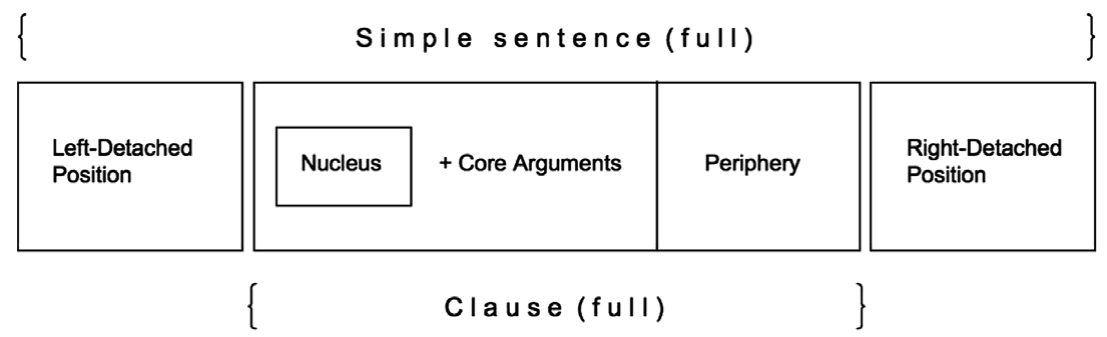

Figure 1. The structure of a clause represented schematically.

may be a verbal or a nominal of some sort, defines the NUCLEUS, which is the main syntactic constituent in the clause. The arguments of the predicate are syntactically CORE ARGUMENTS which, together with the nucleus, constitute the clausal CORE; non-arguments (e.g., units with an adverbial function) form the PERIPHERY.

The core plus periphery structure of the clause may be complemented with several core-external and clause-external elements. Typical core-external (yet clauseinternal) elements include question words that, depending on the language, occur either in PRE-CORE SLOTS or POST-CORE SLOTS. Typical clause-external elements, in turn, are topics and tags. Of these, the former tend to occur in the LEFT-DETACHED POSITION whereas the latter usually occupy the RIGHT-DETACHED POSITION. The most notable difference between pre- and post-core slots and left- and right-detached positions is that the detached positions are separated from the clause by an intonation break or a pause (Van Valin \& LaPolla 1997, Van Valin 2005).

The clause always constitutes a simple SENTENCE that may, at least theoretically, consist of all of the above-mentioned elements. Such a structure, without pre- and post-core slots, is displayed schematically in Figure 1 (Jantunen 2007, following Van Valin \& LaPolla 1997). Linear and/or hierarchical combinations of two or more clauses are treated as COMPLEX SENTENCES (see Section 3.4).

\subsection{Clauses with a verbal nucleus}

So far, the focus of syntactic research into FinSL has been on finding out the order of the core elements in declarative transitive clauses (Jantunen 2008; see also AlaSippola 2012). The main finding of the work has been that these clauses are not always ordered in exactly the same way, but can display at least three structural combinations of the verbal nucleus (V) and its two core arguments, the A ARGUMENT (the argument referring to the more active, typically agentive participant in a transitive situation) and the P ARGUMENT (the more passive, typically patient-like argument). These orders are AVP, APV, and PAV. The type of clause has been found to affect sign order in that isolated clauses only occur with the orders AVP and APV, whereas clauses extracted from longer stretches of discourse may also manifest the order 
PAV. However, even in discourse settings, the order PAV seems to be highly marked (see Ala-Sippola 2012). The orders AVP, APV, and PAV are demonstrated in (2a), (2b) and (2c), respectively (data from Jantunen 2008) (for coding and transcription conventions, see Appendix).

(2) a. ${ }[\mathrm{WOMAN}]_{\mathrm{V}}[\mathrm{TO}-\mathrm{VIDEOTAPE}]_{\mathrm{P}}[\mathrm{MAN}]$

'A woman videotapes a man.'

b. ${ }_{\mathrm{A}}[\mathrm{GIRL}]_{\mathrm{P}}[\mathrm{TV}]_{\mathrm{V}}[\mathrm{LOOK}-\mathrm{AT}]$

'A girl watches television.'

c. ${ }_{\mathrm{P}}[\mathrm{BOOK}]_{\mathrm{A}}[\mathrm{ME}]_{\mathrm{V}}[\mathrm{SEARCH}] /$ FIND / SIGH-OF-RELIEF / ...

'I was looking for a book, and fortunately I found it. ...'

Sign order in FinSL transitive clauses has been shown (Jantunen 2008) to exhibit regular patterns similar to those found in other sign languages (see Liddell 1980 for American Sign Language, Engberg-Pedersen 2002 for Danish Sign Language, Johnston \& Schembri 2007 for Australian Sign Language). For example, an A argument is always expressed before $\mathrm{V}$, and there is also a strong tendency to express an $\mathrm{A}$ argument before a $\mathrm{P}$ argument. Moreover, verbal-initial structures are not used, and the gesturally DEPICTIVE Type 3 verbals (Jantunen 2008, 2010) including a classifier morpheme (e.g., CL-G-'move towards'-2-1 'a person walks towards') are placed at the end of the utterance (see example (11) below); this applies to all structures containing Type 3 verbals. Otherwise, the type of verbal - that is, whether the verbal belongs to the PLAIN Type 1 (e.g., THINK) or to a gesturally INDICATING Type 2 (e.g., GIVE) - does not affect the sign order (Jantunen 2008, 2010; Ala-Sippola 2012), as has been claimed for other sign languages (e.g., Kegl 2004 for American Sign Language). As the typology of FinSL verbals is relevant to the discussion of ellipsis, the main characteristics of the three verbal types are summarized in Table 1, taken from Jantunen (2008). ${ }^{2}$

Intransitive clauses in FinSL all follow the scheme SV (where S refers to the single, typically agentive participant in the situation encoded by an intransitive verbal). Examples of isolated FinSL intransitive clauses are given in (3) (data from Takkinen, Jantunen \& Ahonen 2010).

(3) a. $\left.s^{[B A B Y}\right]_{v}[$ SLEEP $]$

'A baby sleeps.'

b. s[COUPLE] $\mathrm{v}[\mathrm{DIVORCE}]$

'The couple divorced.'

The fact that FinSL places the main argument in front of the verbal in both transitive and intransitive clauses suggests that FinSL is a more nominativeaccusative type of system than absolutive-ergative, as has sometimes been suggested (Itkonen 2001). This interpretation is also supported by the observation that if core arguments are to have any markers, they attach only to $\mathrm{P}$ and not to A or S. An 


\begin{tabular}{|c|c|c|c|c|c|}
\hline & Example & $\begin{array}{l}\text { LOVE, KNOW, } \\
\text { and LIKE }\end{array}$ & $\begin{array}{l}\text { LOOK-AT, TEACH, } \\
\text { and ASK }\end{array}$ & $\begin{array}{l}\text { CL-G-EXIST- } \\
\text { 'location' 'upright } \\
\text { person is at some } \\
\text { place' }\end{array}$ & $\begin{array}{l}\text { CL-F-'move in careful manner' } \\
\text { 'to move a pea-like tiny object } \\
\text { in a careful manner', and } \\
\text { CL-Y-'fly in a whirling } \\
\text { manner' 'an airplane flies in a } \\
\text { whirling manner' }\end{array}$ \\
\hline
\end{tabular}

Table 1. FinSL verbal types (Jantunen 2008). 
example of a context where this may happen is when $\mathrm{P}$ has the semantic role of a recipient; in such cases the $\mathrm{P}$ argument may be affixed, for example, with a palm-up pointing that is directed towards the physical or abstract location of the entity with the role of the recipient (Jantunen 2008). In general, however, concanative morphology seems not to be used significantly to mark participant roles in FinSL (Jantunen 2008, Ala-Sippola 2012).

\subsection{Clauses with a nominal nucleus}

Clauses with an identifying nominal predicate - that is, equative clauses (e.g., The capital of Finland is Helsinki) - have also been investigated in FinSL (Jantunen 2007, 2009). This work has shown that FinSL uses no copula between the nonpredicating and predicating NPs. However, this finding may be slightly distorted because many equative expressions can occur with a near copula-like element, the so-called PI sign, in front of the predicating NP. This sign is probably in the process of grammaticalization into a copula, but at the moment it is used signer dependently as a modal device expressing certainty. Equative clauses with and without PI are demonstrated in (4) (data from Jantunen 2007).

(4) a. NP-non-pred $\left[\right.$ HIS SURNAME] NP-pred $[\text { LAKE-n-e-n }]^{3}$

'His family name is Järvinen.'

b. NP-non-pred[FRANCE OWN CAPITAL] PI NP-pred[PARIS]

'The capital of France is Paris.'

Two juxtaposed NPs also form the basic structure of other nominal clause types in FinSL (see Rissanen 1998). These include characterizing and locative clauses, demonstrated in (5a) and (5b), respectively. The characterizing clause in (5a) may be produced with an optional PI, which further suggests that the sign may be in the process of grammaticalizing into a copula.

(5) a. NP-non-pred[GIRL] NP-pred[CLEVER]

'The girl is clever.'

b. NP-non-pred [OWN WORK-PLACE] NP-pred[HERE]

'My workplace is here.'

In (5a), the property-assigning sign CLEVER is treated as a nominal, not as an adjective. ADJECTIVE is not an independent word class in FinSL. All signs denoting properties are analyzed semantically and grammatically either as marginal NOMINALS or marginal VERBALS, the two forming the two major word classes in FinSL (Jantunen 2010). For example, signs for color (e.g., RED, GREEN) are classified as nominals: they refer to abstract 'color entities', they cannot display event structure or information related to aspect in their form (a major characteristic of verbals), and they can occur at the beginning of structurally minimal declarative transitive clauses. In 
contrast, signs expressing feelings and emotional states (e.g., HAPPY, ANGRY) are typically analyzed as verbals: they express states (i.e. they are stative verbals), they can encode information related to the structure of the event in their form (e.g., 'be happy for a long time'), and, like other verbals, they cannot be the first constituents in structurally minimal declarative transitive clauses.

Further support for the non-existence of the word class adjective as a grammatical category in FinSL is provided, for example, by the identical morphosyntactic marking of both 'genitive attributes', as in (6a), and 'adjective attributes', as in (6b), and predicating core verbals, in (7a), and predicating property signs, in (7b) (data from Takkinen et al. 2010):

(6) a. [BOY APPLE] 'boy's apple'

b. [RED APPLE] 'red apple'

(7) a. GIRL CRY 'Girl cries.'

b. GIRL HAPPY

'Girl is happy.'

In the analysis of nominal clauses (such as the equative clauses in (4) or the characterizing clause in (5a)), the question of the syntactic status of the first nonpredicating NP has always been problematic (Kelomäki 1998). The problem arises from the fact that this NP cannot be properly counted either as a core argument (because there is no verbal whose semantics would require its presence) or as a peripheral element (because the NP does not straightforwardly set the context). In FinSL research, the issue has been solved by treating the non-predicating NP simply as a nominal-structure-specific core-internal element (Jantunen 2007).

The main evidence for this core-internal treatment comes from the distribution of question words: because question words occur either in pre- or post-core positions, and because, for example, in (4a) the question word WHY would come before the first non-predicating NP, the NP must be counted as core-internal. Such an analysis is given in (8).

content question operator

[WHY CORE[HIS SURNAME LAKE-n-e-n]]

'Why his family name is Järvinen?'

In (8), the clause is layered with a non-manual interrogative operator for content questions. The primary features of this question operator are furrowed eyebrows and a forward head tilt. There are specific non-manual operators also for polar questions and for negation. In the main polar question marker, the eyebrows are raised and the 
head tilts forward. The primary negative operator is a headshake (see Rissanen 1985, Jantunen 2003, Savolainen 2006).

\subsection{The topic-comment structure}

Clauses in FinSL may be preceded by a left-detached topic NP that sets an interpretative (e.g., spatial, temporal, or individual) framework for the following main predication (comment), expressed by the clause (see Figure 1). It has been argued that topics in FinSL are marked syntactic-prosodically (i.e., by their sentence-initial position, pause, and a non-manual feature, 'eyes widened and eyebrows raised') and sometimes morphologically (i.e., indexically with a pointing) (Jantunen 2009). Overall, it has been claimed that FinSL topic structures resemble those found in topicprominent languages, for example, in Mandarin Chinese (Chafe 1976). Examples of topic structures with spatial and temporal topics are given in (9a) and (9b), respectively (data from Jantunen 2009).

(9) a. non-manual topic marker TOP[NIGHT CLUB INDEX-3] / ME WORK DOORMAN

'As for that night club (there), I work as a doorman.'

b. non-manual topic marker TOP[NOW EVENING] / ME GO-TO PUB

'As for this evening, I will go to the pub.'

If spatial or temporal information is not expressed as a topic, it can be expressed within the clause as a periphery. It seems that especially in discourse settings (and depending, for example, on the clause type) the position of peripheral elements within the clause can vary. However, perhaps in order to maximize the difference between topic-comment structures and plain clauses, there is a tendency to express the peripheral information at the end of the clause, as in (10).

\section{(10) MY SON BORN PERIPHERY[TWO-THOUSAND FIVE]}

'My son (was) born in two thousand and five.'

In FinSL, utterances containing descriptive Type 3 verbals (see Table 1) are typically topic-comment structures. Syntactically, these verbals occur in sentencefinal position and can be considered to be full, well formed clauses (Jantunen 2008). The analysis stems partly from the fact that the verbals include fused classifier handshape(s) that can be analyzed as nominal core arguments of the predicate (Van Valin \& LaPolla 1997, Dixon \& Aikhenvald 2000, Van Valin 2005). Another reason for the clausal interpretation is the fact that the meaning of these verbals is typically very clause-like, that is, it covers the whole event. However, due to the semantics of classifiers, there tends to be some vagueness in the meaning of such verbals/clauses. This can be overcome by the use of contextual information or by 
linguistic specification. The specification means adding a full NP in front of the verbal/clause as a topic, as in (11). Because descriptive Type 3 verbals may need the specification of, for example, location (i.e., ground), or may include two different classifiers (e.g., ground+figure) (i.e., they can contain representational activity in both hands), FinSL is considered to be able to have chains of at least two consecutive topics.

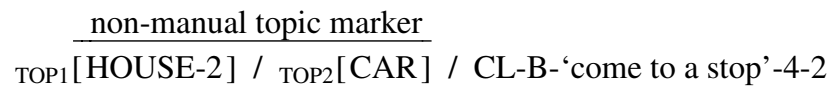

'Car (literally: rectangular object) stops near the house.'

Topics have an important distributional restriction as far as non-manual activity is concerned: they cannot be layered with negative or interrogative operators. This restriction stems partly from the general function of topics: there is no point in denying the framework required in the interpretation of the comment, nor in using a question as such a framework (see Kroeger 2004). The restriction can also be motivated by the fact that there is already a non-manual (topic) marker present in topics.

\subsection{Other complex structures}

Adding a topic before the clause makes the structure as a whole more complex. Another way to create complex structures is to bind together two (or more) clauses. Structurally, the resulting complex sentence can be analyzed as being constructed either through coordination or through subordination. The exact mechanisms involved in these processes have not yet been fully researched in FinSL but, as evidenced by the examples in (12), complex sentences created with both strategies definitely exist.

\section{(12) a. BOY GO-TO SCHOOL ' BUT GIRL STAY-AT-HOME}

'The boy went to school but the girl stayed at home.'

\section{b. ME THINK ' GIRL SMART}

'I think that the girl is smart.'

The sentence in (12a) is an example of a two-clause coordinated structure. In the structure, both clauses are of equal status, which is evidenced by the fact that the positions of the clauses can be changed without any change in the overall meaning (Johnston \& Schembri 2007). In the example, the clauses are conjoined with the sign BUT, which belongs to the minor word class of conjunction signs in FinSL. If the sign BUT is left out of the sentence, the meaning of the sentence changes to 'The boy went to school and the girl stayed at home'. This suggests that the basic coordination strategy in FinSL is simply juxtaposing two clauses.

In (12b), the two clauses do not have equal syntactic status. Instead, the first clause is the main clause and the second clause is the subordinate clausal complement (core argument) of the verbal THINK, the nucleus of the main clause. The verbal 
THINK itself is ambitransitive in FinSL, that is, it can be used either intransitively or transitively (Dixon \& Aikhenvald 2000). In the present context, it is being used transitively.

Yet another way to create syntactically - and semantically - complex sentences in FinSL is to use blends. As a term, BLENDING was introduced to mainstream sign language linguistics by Liddell (2003), who used it in his cognitively oriented framework to capture certain very common referential and pantomime-like phenomena in signing. An example of the first type - of the complex referential use of space - is given in (13). In the example, the signer exploits the topic-comment structure and creates a blend - a 'conceptual mixture' - of (primarily) a referential token space (the referential locations for 'cat" and ' $\mathrm{dog}^{\text {') }}$ and the real space (the physical reality where the signer exists and signs) to represent the semantic event 'the dog attacks the cat'.

(13) DOG INDEX-3 token-a $_{\text {a }}$ CAT INDEX-4 $4_{\text {token-b }}$ / DOG ATTACK-3-4 CAT

(After establishing the tokens:) 'The dog attacks the cat.'

An example of a more pantomime-like blend is given in (14), from Lukasczyk (2008). This is an example of the so-called surrogate blend, which means that the signer himself comes to represent the entity (mouse) about which he first starts to sign. The blend in this case is manifested through an extensive use of non-manual articulators (eyes, head, body) mimicking the in situ habitus of the mouse.

(14) In a story in which the Fox has just asked the question 'Who lives there?':

\section{surrogate}

.. . / INDEX-3 $3_{\text {token }}$ MOUSE GNAWLER SAY / ME-3

'The mouse Gnawler answered: "Me".'

In the blend part of (14), the manual activity (the production of the sign ME in the tokenized location of the mouse) represents the words of the mouse, not those of the signer/protagonist. In fact, in FinSL, surrogate blends are a natural way to report direct speech, that is, the words and thoughts and actions of others (Lukasczyk 2008). In general, as surrogate blends combine syntactic structure with pantomimelike activity, they also demonstrate the fuzziness of the border between language and gesture in sign languages (for more, see Liddell 2003).

\section{WHAT CORE-INTERNAL ELEMENTS CAN BE LEFT OUT IN FINSL CLAUSES?}

The previous section described the structure of well formed, complete clauses in FinSL. However, as ellipsis is documented as being very frequent in FinSL, clauses are 
very often incompletely realized in everyday communication. This section describes what core-internal elements may be left out of FinSL clauses.

\subsection{Ellipsis of core arguments}

In clauses with a verbal nucleus, a very common form of ellipsis is the elision of the S/A argument (Wulf et al. 2002, Ala-Sippola 2012). Several examples of clauses with a missing S/A are given in (15). The examples demonstrate how the elided S/A element can be either a pronoun, as in $(15 a, b)$, or a full NP, as in (15c, d). Moreover, the examples show how the missing element may be accessible either pragmatically, (15a-c), or syntactically, (15d). (From now on, clauses in focus are bracketed and their cores are indicated with boldface; the elided element is indicated with the symbol $\varnothing$.)

(15) a. YES YES 'PRECISELY' [Ø REMEMBER (Ø)]

'Yes, yes, that's how it was! (I) remember.'

(Jantunen et al. 2012; dialogue data set 2, participant Jr, clause 11)

b. The signer has talked about the birth of her son in 1999:

... / [TWO-THOUSAND . . ONE Ø MOVE HELSINKI+DIRECTION]

'In two thousand and ... one (we) moved to Helsinki.'

(Jantunen et al. 2012; dialogue data set 2, participant Jr, clause 20)

c. The signer has just mentioned that Swedish is constantly used in Vaasa, a city in Western Finland:

.. . / [Ø SPEAK SWEDISH ALMOST EVERY DAY]

'(People) speak Swedish almost every day.'

(Jantunen et al. 2012; dialogue data set 1, participant Sr, clause 18)

d. MAN GO-IN / [ Ø SEE WOMAN]

'The man goes in and (the man) sees the woman.'

The missing elements in $(15 \mathrm{a}-\mathrm{c})$ have no syntactic antecedents. In (15a), the referent of the elided first person pronoun is understood from the fact that it the signer herself who produces the whole short utterance in response to the other signer's assertion. In (15b), the referent of the missing first person plural pronoun is recovered from the fact that the signer has just talked, among other things, about the birth of her child a few years earlier; the addressee also knows the signer and her family's history personally, so overtly mentioning 'we' would be entirely redundant. In $(15 \mathrm{c})$, the generic human referent of the elided NP is retrieved only on the basis of world knowledge, that is, basically from the commonly known fact that it is people in general who speak languages. ${ }^{4}$

In (15d), the second clause of the coordinated structure lacks an overtly expressed A argument. However, unlike in $(15 \mathrm{a}-\mathrm{c})$, the referent of the missing element has an antecedent in the preceding syntactic context: it is co-referential with the $S$ of the 
first clause, that is, the elided A represents zero anaphora. Superficially, (15d) seems to suggest that the referent of the missing A ('man') is recovered on the basis of a pivot constraint (similar to that which governs the occurrence of ellipsis in corresponding English sentences) and that FinSL has an S/A pivot (Dixon 1994). However, this analysis is contradicted by the fact that it is possible in FinSL to elide also $\mathrm{P}$ arguments in contexts that meet the criteria of an S/P pivot. An example of this is given below in (16).

\section{(16) MAN GO-IN / [WOMAN LOOK-AT Ø]}

'The man goes in and the woman looks at (the man/him).'

In (16), the elided $\mathrm{P}$ of the second clause is also co-referential with the $\mathrm{S}$ ('man') of the first clause. Consequently, because languages follow either the S/A or S/P paradigm (Dixon 1994), the identity of the referents of the missing elements in (15d) and (16) cannot be recovered on the basis of any pivot constraint. Instead, it is suggested that the referential identity of the missing elements is in both cases retrieved on the basis of the discourse theme 'man'. This is a widely used strategy also cross-linguistically (Dixon 1994). More generally, examples (15d) and (16) suggest that FinSL has no syntactic pivot. Engberg-Pedersen (2002) has put forward a similar claim for Danish Sign Language.

If the context is right, it is possible to elide both core arguments of transitive structures at the same time. This can be argued to be the case in (15a), which, in addition to the main argument, lacks also the (optional) second argument. Omissions of both arguments are known to happen relatively frequently also with indicating Type 2 verbals, to be discussed in terms of ellipsis in Section 5.

\subsection{Ellipsis of nuclei}

In addition to core arguments, verbal nuclei too may be elided in FinSL. Two examples demonstrating this are given in (17).

(17) a. The signer is answering the question what languages she uses daily:

[ME Ø SIGN-LANGUAGE] / . . .

'I (use) sign language.'

(Jantunen et al. 2012; dialogue data set 1, participant Sr, clause 9)

b. GIRL HAS-GOT TWO-PIECES / [BOY Ø ONE-PIECE]

'The girl has two and the boy (has) one.'

In (17a), the overt structure of the elliptical transitive clause is similar to the NP plus NP structure found in nominal clauses (see Section 3). However, as the meaning of the clause obviously cannot be 'I am (in) sign language', the clause is interpreted as being elliptical in terms of its verbal nucleus, the sign USE. The meaning of the missing verbal in this case is inferred, again, on the basis of the preceding context: the 
clause is (part of) an answer to the other signer's question as to how many languages the signer uses in her everyday life. In this stretch of conversation the verbal USE occurs only in this discourse-initial question and is consistently omitted in the rest of the discussion.

The example in (17b) presents another type of verbal ellipsis which is referred to in the literature as GAPPING. In gapping, the verbal in the second clause of a coordinated (or comparative) structure is unexpressed (McShane 2005) and its meaning is deduced on the basis of the parallelism between the two clauses. Evidently, this parallelism is quite strong and reliable in (17b): if the verbal nucleus of the first clause were omitted, the interpretation of the meaning of the resulting clause would be 'Two (pieces of) girls'. However, in the actual elliptical clause in (17b), there is no danger of such ambiguity.

\subsection{Ellipsis of non-predicating NPs in nominal clauses}

Leaving out core-internal elements is also possible in nominal clauses in FinSL, as demonstrated in (18). However, as nominal clauses are not governed by the semantic requirements of the predicate in the same way as clauses with a verbal nucleus are, it may not always be possible to determine between true cases of ellipsis and, for example, idiomatic uses of impersonal nominal predicates and other fragments (McShane 2005).

(18) a. The signer has just mentioned that she uses sign language daily:

... EVIDENT / [Ø OBVIOUS] / ME WORK HERE

... '(That's/My use of sign language is) obvious. I work here.'

(Jantunen et al. 2012; dialogue data set 1, participant Sr, clause 10)

b.

MAN COME-IN / [Ø TALL]

'The man comes in. (He) is tall.'

In (18a), the clause in focus is considered to be elliptical in either the core-internal full thematic NP or an anaphoric pronoun referring to the previously discussed 'signer's use of sign language'. The analysis rests on the fact that, although FinSL does not use the pleonastic 'It is ...' structure typical of English, it is possible to add an NP or simply an anaphoric pronoun into the structure. The clause in (18b) is elliptical on the same grounds. However, unlike in (18a), the clause in (18b) makes it almost obligatory for the signer to use a surrogate blend in which he/she takes the role of a perceiver who 'eye-balls' the entering man and his height. In the blend, the signer turns his/her head and eyes upwards at the same time as he/she articulates the sign TALL. In the sign, the dominant hand moves vertically upwards with an open palm handshape and a slowly extending wrist. 
Apart from (18b), none of the examples discussed above have included any marked form of non-manual activity that could affect the elliptical reading of the examples. In other words, no example has involved, for example, the substitution of a manually expressed pronominal core argument with a non-manually expressed one. This is a known process in FinSL and does not represent true ellipsis, because the pronoun is present, albeit non-manually. For example, in (19), the $\mathrm{S}$ argument of the nucleus COME-HERE is expressed non-manually with a head nod towards the addressee in between the last two signs of the clause and is, thus, not elided.

content question operator

nod towards the addressee

HOW-MANY YEAR BEFORE COME-HERE

'How many years ago did you come here?'

(Jantunen et al. 2012; dialogue data set 2, participant Jr, clause 2)

In conclusion, this section has shown that it is possible to elide all core-internal clausal material in FinSL: core arguments (S/A and P), core-internal NPs, and nuclei. In the next section, we will outline several grammatical contexts that specifically seem to favor the ellipsis of these elements.

\section{GRAMMATICAL CONTEXTS THAT FAVOR ELLIPSIS IN FINSL CLAUSES}

As seen in the previous section, in many cases ellipsis in FinSL is not conditioned purely grammatically, but its occurrence depends upon textual, pragmatic, sociolinguistic, and other factors. There are, nevertheless, cases of ellipsis in which the motivation to not express something overtly within the clause can be traced back also to a specific grammatical context or structure. This section discusses some of these cases and structures.

\subsection{Question-answer pairs}

One grammatical context in which frequent use of elliptical clauses has been identified in FinSL is that of questions and answers: questions permit reduced answers because all the necessary information is typically contained already in the question part (McShane 2005). An often cited example in the literature is the signed question Are you deaf?, which may be answered only with a head nod (e.g., Rissanen 1985, Jantunen \& Takkinen 2011). Another example, extracted from an actual dialogue, is given in (20) (see also 17a). In (20), the reduced answer contains neither A nor P (the second of which may be analyzed to be a full subordinate clausal complement) because their meaning can be directly inferred from the preceding question. 
Question:

AT-ALL YOU THINK / HOW MANY DIFFERENT LANGUAGES question operator

DURING-ONE-DAY YOU USE

'Have you ever thought how many different languages you use during one day?'

Answer:

YES / [Ø OFTEN THINK [Ø]]

'Yes, (I have) often thought (about it/how many languages I use daily).'

(Jantunen et al. 2012; dialogue data set 1, clauses 1-3)

Cross-linguistically, question-answer context is not always considered to be a proper domain from which ellipsis should be identified as it may be governed by a specific set of interactional and grammatical rules (McShane 2005). However, it is claimed here that the case is different with FinSL: the question-answer set-up in FinSL is not limited to traditional interrogative dialogue situations but it is used also for other purposes, for example, in monologues to establish discourse focus.

\subsection{Two-clause coordinated structures}

Another grammatical context clearly favoring ellipsis in FinSL (and in other languages; McShane 2005) is the two-clause coordinated structure. In these structures, it is normally the second clause from which some material is elided. The two clauses do not need to have similar structures (see (15d) and (16)) but if they do, like the clauses in the first sentence of (21), the structural parallelism between the two clauses provides an extra cue (in addition to the cue provided by the discourse theme) to recover the referential identity of the elided core arguments (McShane 2005).

(21) The signer is talking about his journey abroad:

SUCH-AND-SUCH / ME FLY-THERE ' [Ø STAY-THERE] / Ø MEET THERE / DALLAS d-a-1-l-a(-s) AIRPORT THERE / Ø MEET . . .

'So, I flew there and stayed there. (I) met there, there at Dallas airport... (I) met there (the person responsible for international affairs.)'

(Jantunen 2008)

The clause in focus in (21) contains a typical example of a zero anaphora in a coordinated structure. The omitted element is the discourse theme ('me') with a syntactic $\mathrm{S}$ function and it is inferred from the parallel thematic $\mathrm{S}$ in the antecedent clause. This particular example is a part of a longer stretch of signing and, as illustrated in (21), the discourse theme is omitted also in subsequent clauses. Overall, there are three consecutive clauses in (21) that occur without the S/A argument. 
The examples in (22a) and (22b) illustrate yet other types of ellipses typical of coordinated structures and FinSL, namely the elision of verbal nuclei (and related elements) in the second clause. Sentence (22a) is an example of gapping, similar to that discussed in (17b). Sentence (22b), on the other hand, is an example of either stripping or VP ellipsis. In STRIPPING, the elliptical process strips away all but one main constituent in the ellipsis clause under identity with the antecedent clause (some less important constituents may remain intact as well), whereas in VP ELLIPSIS the verbal phrase (the verbal, its possible $\mathrm{P}$ argument, and periphery) are elided (McShane 2005). The analytical difficulty in (22b) concerns the syntactic status of the sign ALSO: Is it a less important constituent left intact (stripping) or an element outside the scope of the VP (VP ellipsis)? The fact that VP ellipsis is typically co-licensed with a lexical item (e.g., auxiliaries in English, see McShane 2005) suggests the VP ellipsis reading: in (22b), the ellipsis is not possible if the sign ALSO is not present.

(22) a. Boy and girl go to the shop together:

\section{BOY BUY APPLE' [GIRL Ø ORANGE]}

'The boy bought an apple and the girl (bought) an orange.'

b. Boy and girl go to the shop together:

\section{BOY BUY APPLE' GIRL Ø ALSO}

'The boy bought an apple and the girl (bought an apple) too.'

Verbal related elision phenomena in coordinated structures typically rely heavily on the structural parallelism between the two conjoined clauses (see (17b) and (22)). However, as demonstrated by (23), this does not always have to be the case. In (23), the verbal related ellipsis known as SLUICING is not primarily licensed by the structural parallelism but by a $w h$-word; the elision process itself deletes the relevant interrogative phrase (McShane 2005).

content question operator

ME ALREADY-KNOW YOU BUY+ALREADY APPLE' BUT WHY [Ø]

'I know that you bought an apple but (I don't know) why (you bought it).'

\subsection{The topic-comment structure}

In addition to two-clause coordinated structures, another syntactic configuration clearly favoring ellipsis in FinSL is the topic-comment structure. In the topiccomment structure, it is the comment clause that is often incomplete. In the retrieval of the referents of the elided elements, both textual and contextual knowledge are used extensively. For example, in (24), it is obvious from the discourse context that it is the signer HERSELF who signs at home and at the university campus because SHE has just been talking about HER OWN daily use of languages. A deeper underlying factor that probably further enables the inference of the elided elements in topic-comment 
structures is our universal ability to make compositional semantic associations (see Gil 2008). In other words, topic-comment constructions, including the elliptical one in (24), are in the end understood on the basis of simply putting together, bit by bit in the given context, the basic meanings of the main constituents that have been expressed.

(24) When explaining the daily use of languages from a personal perspective:

non-manual topic marker

AT-HOME / UNIVERSITY AREA / cOM[Ø SIGN]

'As for my home, as for the university campus, (I) sign.'

(Jantunen et al. 2012; dialogue data set 1, participant Jr, clause 12)

In topic-comment structures in which the topic resembles either the S/A or $\mathrm{P}$ argument, ellipsis is almost obligatory. Examples of such structures are given in (25); in (25a), the topic is a unit resembling an A argument whereas in (25b) it resembles a $\mathrm{P}$ argument.

(25) a. non-manual topic marker

OWN BAPTISE AUNT / COM[Ø LIVE SWEDEN+DIRECTION]

'As for my godmother, (she) lives in Sweden.'

(Jantunen 2009)

b. non-manual topic marker

KEY + INDEX-2down / COM[WOMAN Ø TAKE-2down]

'As for the key, a woman picks (it) up.'

(Jantunen 2009)

In the formal framework (e.g., Liddell 1980, ${ }^{5}$ Aarons 1996, Sandler \& Lillo-Martin 2006), the topics in (25a) and (25b) have been analyzed as complements (subjects, objects) moved from their clause-internal position to the clause-external left-detached (or corresponding) position. This view presumes that such topics are still in a semantic and syntactic relation to the predicate/nucleus. In the study of FinSL, the structures in (25) have not been treated in this way. Instead, they have been considered to be independent topic-comment structures that involve no movement of any unit. In other words, the structures in (25) have been claimed to be similar to those in (9), with a frame-setting adjunct-like topic that has no syntactic or semantic relationship to the predicate/nucleus (Jantunen 2008, 2009).

The investigation of ellipsis provides support for the 'no move' analysis of topics in (25), which is preferred in research into FinSL. Although it would be a marked option, it is definitely possible to produce both examples so that their comment clauses include an anaphoric pronoun referring to the referent identified by the clause-external topic (see the symbol $\varnothing$ in the examples). If the topics were moved, they would still have a relationship with the predicate/nucleus, and no pronouns could be used in their respective positions in the comment clause. Consequently, the 
clauses are simply elliptical (and not incomplete due to any movement) and, in fact, represent instances of near obligatory zero anaphora.

\subsection{Blend structures}

Wulf et al. (2002) have shown statistically that the grammatical use of blends (CONSTRUCTED ACTION in their terminology) is often a catalyst for elliptical clausal structures in signed language (see also Johnston \& Schembri 2007). The surrogate blend in (26), taken from Lukasczyk (2008), provides an example of this in FinSL. In (26), the signer begins his narrative with a blend in which he presents the protagonist, who is sitting in a train. He then goes on to report the actions and mental processes of the protagonist by first producing the verbal sign LOOK-AT (directed towards the mentally constructed window). Syntactically, the verbal functions as the transitive nucleus of a clause and could be expected to require an expression of both A and $\mathrm{P}$ core arguments. However, in (26), neither of these is present overtly. Instead, their referents are constructed mentally on the basis of the context created by the blend. In the end, the mental image of the blend is so strong that the only possible way of making sense of the referents is to assume that the agentive one is the protagonist himself and the more patient-like is the generic abstraction 'something outside the window'.

surrogate

[Ø LOOK-AT-outside Ø] COME-INTO-MY-MIND ’ ME LITTLE . . .

'As (I) sat there and looked out the window a memory from my childhood came into my mind...'

(Lukasczyk 2008)

Sentence (27) contains another example of the relationship between a surrogate blend and an elliptical clause. In (27), the argument structure of the transitive predicate $\operatorname{look}(\mathrm{x}, \mathrm{y})$ is once again realized incompletely because the NP P argument - supposed to convey the meaning 'text (on the computer screen)' - is elided. However, from the point of view of semantic interpretation, this is not a problem because the blend of the signer into the protagonist provides all the required information for the construction of the correct meaning. In this case, the blend is manifested through the signer's body posture, eye gaze, and the downward movement of the hand and fingers, which represent the eyes. Overall, the blend explicates the meaning by creating a pantomimic image of the signer actually reading the text from the computer screen.

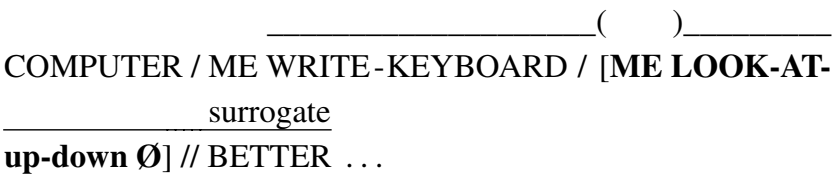


'As I read the (text) that I had written (on the screen) I noticed that [the two paragraphs were better in the opposite order ... ]'

(Jantunen 2008)

\subsection{Type 2 verbals}

One more grammatical context in which ellipsis is clearly favored in FinSL revolves around the use of indicating Type 2 verbals (see Table 1 above). The Type 2 verbals include a gestural component which allows them to be meaningfully directed in space between referents of the situations they encode (Liddell 2003; Jantunen 2008, 2010). As the referents are indicated by the forms of the verbals themselves, their overt expression within the clause is unnecessary by definition.

The locations of the referents may be either physical or grammatically established. In (28a), the Type 2 verbal TEACH is directed between two physically present persons. The clause formed around the verbal does not contain overtly expressed A or P but their referents are easily retrieved from the physical context from the knowledge that the verbal TEACH always points towards the patient-like referent. In (28b), the locations of the referents are first established by means of tokenization (see Section 3). After this, the Type 2 verbal HIT, forming a clause by itself, is directed from the first location to the second one. The overall meaning of the clause is inferred on the basis of the established tokens and the directionality.

(28) a. During a discussion about teaching:

[Ø TEACH-me-you Ø]

'(I) teach (you).'

b. j-u-s-s-i INDEX-3 token-a $_{\text {/ p-e-k-k-a INDEX-4-token-b }}$ [ [Ø HIT-3-4 Ø]

'[After establishing tokens for Jussi and Pekka:] (Jussi) hits (Pekka).'

In everyday communication, directing Type 2 verbals between the locations of referents may not be very common in FinSL (see De Beuzeville, Johnston \& Schembri 2009 for Australian Sign Language). Instead, it may be that the phenomenon is used extensively only in certain textual contexts, such as in formal reporting and in education (Lautala 2012). In these contexts, the primary function of the directionality - and, consequently, also of the ellipsis - may ultimately be to increase textual cohesion (Lautala 2012).

Type 2 verbals have been found to be linked to the phenomenon of blending (see De Beuzeville et al. 2009). Also other contexts favoring ellipsis (blends and Type 2 verbals included) are often realized at the same time. This makes the overall effect of ellipsis sometimes appear very strong as it is possible for the discourse to proceed only with a very minimal amount of lexically expressed information. 


\section{WHAT DOES ELLIPSIS TELL US ABOUT FINSL AND ITS CLAUSES?}

Here it is argued that the relatively high frequency of ellipsis in FinSL and the nature of the ellipsis have certain consequences for the analysis of FinSL and its clauses. First, concerning FinSL in general, the frequent use of ellipsis, together with the elliptical phenomena presented in this paper, suggest that FinSL is a highly discourse-oriented language. According to McShane (2005:18-19), discourse-oriented languages fulfill the following criteria in terms of ellipsis:

- discourse-oriented languages permit more ellipsis than non-discourse-oriented languages;

- in discourse-oriented languages, thematic elements tend to be elided;

- in discourse-oriented languages, ellipsis is not merely an optional grammatical process but an important means of supporting the discourse structure.

With regard to the first criterion, Ala-Sippola (2012) has shown that half of the transitive clauses in her data were produced without A arguments and that this may be a trend in FinSL in general. Although no numerical investigations have been conducted on the omission frequency of other core-internal clausal elements in FinSL ( $\mathrm{S}$ and $\mathrm{P}$ arguments, non-predicating core-internal NPs, nuclei), the study by Wulf et al. (2002), which investigated the combined omission frequency of both $\mathrm{S}$ and $\mathrm{A}$ (as in pro drop) in American Sign Language, indicates that the overall frequency of clause-internal ellipsis in FinSL may be even higher than was found by Ala-Sippola: in Wulf et al.'s study, the total share of clauses that were elliptical in terms of the subject-like core argument was found to be $65 \%$. All in all, the difference between the ellipsis rate in FinSL (and in American Sign Language) and the ellipsis rate in non-discourse-oriented languages such as Spanish (7.5\%; Alcántara \& Bertomeu 2005 ) is notable, and cannot be accounted for simply by any individual grammatical factor.

Concerning the second criterion, the data presented in this paper have clearly indicated that FinSL tends to elide precisely the thematic elements. In particular, the issue was discussed with regard to examples (15d) and (16), which showed that the referents of zero anaphora in FinSL are not recovered with the help of any syntactic pivot constraint but rather by relying on knowledge of the discourse theme. Moreover, example (21) - containing a short travel story - demonstrated how the discourse theme (me) was elided in three consecutive clauses. Such processes are not found in non-discourse-oriented languages such as English.

The final criterion - that ellipsis is not merely an optional grammatical process in discourse-oriented languages but an important means of supporting the discourse structure - has not been explicitly discussed in the present paper. However, the existence of such a feature in FinSL was hinted at during the discussion of Type 
2 verbals in Section 5.5, where it was suggested that the use of these verbals inherently favoring ellipsis - may be restricted only to certain textual contexts (e.g., formal reporting, education) and that their primary function - and also that of ellipsis may be to increase textual cohesion in these contexts (Lautala 2012). More generally, also the fact that zero anaphora is frequently used in FinSL (Ala-Sippola 2012) supports this conclusion: if the use of anaphoric pronouns (instead of a full NP) is to establish coherence by signaling to the addressee that the referent is already familiar and should be found in the preceding discourse context (Hendriks \& Spenader 2005), then zero anaphora must establish coherence even more strongly by assuming that the addressee can retrieve the referent without needing any overtly referring element.

Concerning the unit clause in FinSL, the frequent use of ellipsis - that is, the fact that the core elements in the clause are omitted relatively freely - suggests that the clause in FinSL is not a very stable unit syntactically. This observation may be important for future work on syntax in FinSL: if the basic structure of the clause in FinSL is very unstable to begin with (and involving ellipsis, for example), it may be that the clause should not be given a central role in the description of FinSL syntax. If this line of reasoning is taken to its logical conclusion, it means that in future analysis of FinSL syntax it might prove fruitful to abandon the traditional clause-centered line of thinking altogether and consider FinSL from a whole different perspective.

The idea of departing from traditional clause-based syntax is not a new one in the field of FinSL research, as it has been presented before (on similar grounds) by Jantunen (2008). Superficially, the proposal seems to be empirically grounded and, in the history of linguistics, not completely unheard of (see e.g., Brazil 1995, who argues for the prosody-based linear surface-level analysis of spoken language syntax). However, there are several factors that speak against such a departure. On a practical level, a major one is the fact that no one really has any fully developed, signlanguage-specific alternative to the clause-centered tradition to offer. Consequently, if the only objective is to describe FinSL syntax, the simplest solution is undoubtedly to continue with the clause, despite its syntactic uncoherence.

On an empirical level, a major argument against the proposal to depart from the framework of traditional clause-based syntax is the simple fact that FinSL has complete clauses. These may be used only in restricted and even formal domains such as in education, but they exist, nevertheless. This being the case, it may also be that in the course of the diachronic development of FinSL (as more and more young people are formally educated through it) the role of the clause is becoming reinforced. This is not inevitable, but it is commonly acknowledged that in the course of time grammars typically acquire more conventions (although in specific domains the number of conventions may also be reduced). What the case will be with FinSL, only the future will show. 


\section{CONCLUSION}

To conclude, the present paper has shown that there is a wide variety of elliptical phenomena in FinSL clauses. More specifically, it has been shown that all coreinternal clausal elements may be elided in FinSL and that, although ellipsis in general is widely permitted, there are grammatical contexts which especially favor ellipsis in FinSL (as in other languages too, partially): question-and-answer pairs, two-clause coordinated structures, topic-comment structures, blend structures, and structures containing indicating Type 2 verbals. In theoretical terms, the paper has also shown how research into ellipsis can contribute significantly to our understanding of what kind of a language FinSL is: it has been argued here that FinSL conforms to the main characteristics of a discourse-oriented language, and that FinSL clauses in general are not very stable units syntactically. In future work and analysis on FinSL syntax, both these factors need to be taken into account.

\section{ACKNOWLEDGEMENTS}

The author wishes to collectively thank the numerous people who have contributed to the completion of the present study and paper. Special thanks are due to the three anonymous reviewers of the journal. The study has been supported financially by the Academy of Finland under grant 134433.

\section{APPENDIX}

\section{Coding and transcription conventions used in this paper}

Manual behavior in signs

$\begin{array}{ll}\text { WOMAN } & \begin{array}{l}\text { Signs are referred to, according to the standard convention in } \\ \text { sign language research, as glosses which are to be understood } \\ \text { as rough translations of signs' core meanings. Notation in } \\ \text { capitals. } \\ \text { A hyphen is used when a single sign is glossed with more than } \\ \text { one (English) word. } \\ \text { LOOK-AT }\end{array} \\ \text { KNOW+CONTEST } & \begin{array}{l}\text { Consecutive signs in compound-like structures are indicated by } \\ \text { the 'plus' sign. }\end{array} \\ \text { A gloss followed by a hyphenated number or numbers identifies } \\ \text { a directional sign (i.e., that the sign is either directed to a } \\ \text { certain location or produced at a certain location). Spatial } \\ \text { locations are drawn from Figure A1 (from Rissanen } \\ \text { 1985:18). The numbers may be followed by words (e.g., up } \\ \text { and down) further specifying the location that is pointed at. }\end{array}$




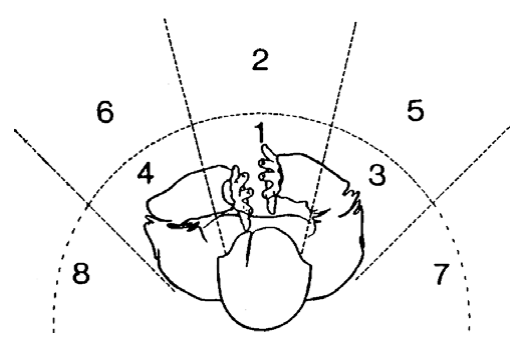

Figure A1. Locations in signing space (from Rissanen 1985:18).

INDEX-3

CL-G-

n-e-n
A non-pronoun pointing made with index finger. As with other signs, the number indicates the relative point the sign is directed towards (see Figure A1).

A $C L$ - 'letter' notation in the beginning of the gloss indicates that the corresponding sign contains a classifier handshape. Handshape symbols are based on Rissanen (1985:68-69).

- 'come to a stop'-4-1 The end part of the gloss in Type 3 signs describes the signs' movement. The words between the quotation marks describe the overall manner of the movement. Numbers indicate the change in the location of the hand in the signing space (see Figure A1).

Letters separated by hyphens refer to fingerspelled words in which every letter is expressed.

Non-manual/temporal behavior in signs

/ Pause.

// A long pause.

, A change in non-manual behavior without a pause (e.g., when signalling a phrase or clause boundary).

The scope of layered grammatical non-manuals (e.g., operators, topic markers, non-manuals used in the construction of surrogate blends) is signalled with a line above the glossing.

\section{Abreviations relating to the analysis}

$\varnothing$

[OWN WORK-PLACE] Syntactic constituents (phrases, clauses, etc.) may be indicated within square brackets. The initial bracket may be preceded by additional grammatical information (see below).

V

Verbal sign. 
$\mathrm{A} / \mathrm{S}$

$\mathrm{P}$

NP-pred/non-pred

TOP

$\mathrm{COM}$

(token)
A unit referring to the single (S) participant of an intransitive event or to the more active (A) participant (prototypically the agent) in the situation encoded by a transitive verbal encoded by an intransitive or transitive verbal, respectively.

A unit referring to the more passive participant in the situation encoded by a transitive verbal (prototypically the patient).

The predicating/non-predicating NP in clauses with a nominal nucleus.

Topic. If there are more than one topic, the topics are numbered.

Comment (clause).

The subscript used in the identification of indexes that establish tokens. Multiple tokens are distinguished by letters.

\section{NOTES}

1. A PIVOT CONSTRAINT controls the way two clauses combine to form a complex sentence; it involves co-occurence and omission conditions on the syntactic functions (S/A and P) of an NP that is common to the two clauses (see Appendix for abreviations relating to the analysis). Pivot constraints are language-specific. See Dixon (1994) for further details.

2. FinSL signs are grouped into two broad lexical-grammatical categories, NOMINALS and VERBALS (Rissanen 1998, Jantunen 2010). The category of nominals include traditional nouns and the category of verbals traditional verbs; in addition, both categories contain lexemes that many languages categorize as adjectives (see Section 3.2). In this paper, the terms nominal and verbal are used also as general typological notions, that is, also when referring to the lexical-grammatical categories of languages other than FinSL.

3. Lake translates into Finnish as järvi.

4. The clause in (15c) may be considered to present a more complex case of ellipsis. Although the semantics of the ambitransitive verbal SPEAK definitely requires the syntactic expression of S or A, it may be that the signer is still deliberately trying to defocus the agent in the clause. If this is the case, (15c) would also involve semantic ellipsis, and could perhaps also be analyzed as a type of passive construction (Shibatani 1985). However, as little is known about passives in sign languages (see Ala-Sippola 2012), and because the focus of the present paper is only on syntactic ellipsis, this analysis is not pursued further here.

5. Liddell (1980) analyzes topic differently from Liddell (2003), the latter of which is used as the main framework in the analysis of blend structures in the present paper. In Liddell (2003), a topic is just a topic, that is, Liddell's (2003) analysis corresponds closely to that used in FinSL research.

\section{REFERENCES}

Aarons, Debra. 1996. Topic and topicalization in American Sign Language. Stellenbosch Papers in Linguistics 30, 65-106. 
Ala-Sippola, Sanna. 2012. Agentin ilmaiseminen suomalaisella viittomakielellä tuotetussa asiatekstissa [Expressing agent in a formal FinSL narrative]. MA thesis, Department of Modern Languages, University of Helsinki.

Alcántara, Manuel \& Nuria Bertomeu. 2005. Ellipsis in spontaneous spoken language. In Spenander \& Hendriks (eds.), 1-10.

Brazil, David. 1995. A Grammar of Speech. Oxford: Oxford University Press.

Chafe, Wallace L. 1976. Givenness, contrastiveness, defineteness, subjects, topics, and point of view. In Charles N. Li (ed.), Subject and Topic, 25-55. New York: Academic Press.

De Beuzeville, Louise, Trevor Johnston \& Adam Schembri. 2009. The use of space with indicating verbs in Auslan: A corpus-based investigation. Sign Language \& Linguistics 12(1), 53-82.

Dixon, R. M. W. 1994. Ergativity. Cambridge: Cambridge University Press.

Dixon, R. M. W. 2010. Basic Linguistic Theory, vol. 1: Methodology. Oxford: Oxford University Press.

Dixon, R. M. W. \& Alexandra Y. Aikhenvald. 2000. Introduction. In R. M. W. Dixon \& Alexandra Y. Aikhenvald (eds.), Changing Valency: Case Studies in Transitivity, 1-29. Cambridge: Cambridge University Press.

Dryer, Mathew S. 2001. What is Basic Linguistic Theory? http://linguistics. buffalo.edu/people/faculty/dryer/dryer/blt (accessed 30 May 2013).

Dryer, Mathew S. 2006. Descriptive theories, explanatory theories, and Basic Linguistic Theory. In Felix K. Ameka, Alan Dench \& Nicholas Evans (eds.), Catching Language: Issues in Grammar Writing, 207-234. Berlin: Mouton de Gruyter.

Engberg-Pedersen, Elisabeth. 2002. Grammatical relations in Danish Sign Language: Topic and subject. In Anneli Pajunen (ed.), Mimesis, Sign, and the Evolution of Language (Publications in General Linguistics 3), 5-40. Turku: University of Turku.

Gil, David. 2008. How complex are isolating languages? In Matti Miestamo, Kaius Sinnemäki \& Fred Karlsson (eds.), Language Complexity: Typology, Contact, Change, 109-131. Amsterdam: John Benjamins.

Givòn, Talmy. 2001. Syntax, vol. 1. Amsterdam: John Benjamins.

Halliday, Michael A. K. 1994. An Introduction to Functional Grammar, 2nd edn. London: Edward Arnold.

Hendriks, Petra \& Jennifer Spenader. 2005. Why be silent? Some functions of ellipsis in natural language. In Spenander \& Hendriks (eds.), 29-38.

Huang, Yan. 2000. Anaphora: A Cross-linguistic Study. Oxford: Oxford University Press.

Itkonen, Esa. 2001. Maailman kielten erilaisuus ja samuus [The difference and similarity of world's languages] (Publications in General Linguistics 4), 2nd edn. Turku: University of Turku.

Jantunen, Tommi. 2003. Johdatus suomalaisen viittomakielen rakenteeseen [An introduction to FinSL linguistics]. Helsinki: Finn Lectura.

Jantunen, Tommi. 2007. The equative sentence in Finnish Sign Language. Sign Language \& Linguistics 10(2), 113-143.

Jantunen, Tommi. 2008. Fixed and free: Order of the verbal predicate and its core arguments in declarative transitive clauses in Finnish Sign Language. SKY Journal of Linguistics 21(2008), 83-123.

Jantunen, Tommi. 2009. Tavu ja lause: tutkimuksia kahden sekventiaalisen perusyksikön olemuksesta suomalaisessa viittomakielessä [Syllable and sentence: Studies on the nature of two sequential basic units in FinSL] (Jyväskylä Studies in Humanities 117). Jyväskylä: University of Jyväskylä. 
Jantunen, Tommi. 2010. Suomalaisen viittomakielen pääsanaluokat [The main parts of speech in FinSL]. In Tommi Jantunen (ed.), Näkökulmia viittomaan ja viittomistoon [Perspectives on sign and lexicon], 57-78. Jyväskylä: University of Jyväskylä.

Jantunen, Tommi, Birgitta Burger, Danny De Weerdt, Irja Seilola \& Tuija Wainio. 2012. Experiences collecting motion capture data on continuous signing. In Onno Crasborn, Eleni Efthimiou, Evita Fotinea, Thomas Hanke, Jette Kristoffersen \& Johanna Mesch (eds.), Proceedings of the 5th Workshop on the Representation and Processing of Sign Languages: Interactions between Corpus and Lexicon, 75-82. Paris: ELRA. [Workshop organized as a part of LREC2012, Istanbul, Turkey, 27 May, 2012.]

Jantunen, Tommi \& Ritva Takkinen. 2011. Puraistaan kieltä 4: Nyökkäys kohti kieltä [lit.: Let's bite the language 4: A nod against the language]. Puhe ja kieli 31(4), 135.

Johnston, Trevor \& Adam Schembri. 2007. Australian Sign Language: An Introduction to Sign Language Linguistics. Cambridge: Cambridge University Press.

Kegl, Judy A. 2004. ASL syntax. Sign Language \& Linguistics 7(2), 173-206.

Kelomäki, Tapani. 1998. Ekvatiivilause [Equative clause]. Helsinki: SKS.

Kroeger, Paul R. 2004. Analyzing Syntax: A Lexical-Functional Approach. Cambridge: Cambridge University Press.

Lautala, Saara. 2012. Kieliopilliset sidoskeinot kahdessa suomalaisella viittomakielellä tuotetussa asiatekstissä [Grammatical cohesion devices in two text documents of Finnish Sign Language]. MA thesis, Department of Languages, University of Jyväskylä.

Liddell, Scott K. 1980. American Sign Language Syntax. The Hague: Mouton Publishers.

Liddell, Scott K. 2003. Grammar, Gesture, and Meaning in American Sign Language. Cambridge: Cambridge University Press.

Lukasczyk, Ulrika. 2008. SANOTTUA, AJATELTUA JA TEHTYÄ. Referointi kolmessa suomalaisella viittomakielellä tuotetussa fiktiivisessä kertomuksessa [Said, thought, and done: Refereeing in three fictive FinSL narratives]. MA thesis, Institute of Teacher Education, University of Jyväskylä.

McShane, Marjorie J. 2005. Theory of Ellipsis. Oxford: Oxford University Press.

Rissanen, Terhi. 1985. Viittomakielen perusrakenne [The basic structure of sign language] (Publications of the Department of General Linguistics, University of Helsinki No. 12). Helsinki: University of Helsinki.

Rissanen, Terhi. 1998. The categories of nominals and verbals and their morphology in Finnish Sign Language. Licentiate thesis, Department of Finnish and General Linguistics, University of Turku.

Sandler, Wendy \& Diane Lillo-Martin. 2006. Sign Language and Linguistics Universals. Cambridge: Cambridge University Press.

Savolainen, Leena. 2006. Interrogatives and negatives in Finnish Sign Language: An overview. In Ulrike Zeshan (ed.), Interrogative and Negative Constructions in Sign Languages, 284-302. Nijmegen: Ishara Press.

Shibatani, Masayoshi. 1985. Passives and related constructions: A prototype analysis. Language 61(4), 821-848.

Takkinen, Ritva, Tommi Jantunen \& Outi Ahonen. 2010. Finnish Sign Language. Ms., University of Jyväskylä.

Spenander, Jennifer \& Petra Hendriks (eds.). 2005. Proceedings of the Cross-modular Approaches to Ellipsis. Edinburgh: Heriot Watt University. [Workshop organized as part of the 17th European Summer School on Logic, Language and Information (ESSLLI 2005), 8-12 August 2005, Edinburgh, Scotland.] 
Van Valin, Robert D. 2005. Exploring the Syntax-Semantics Interface. Cambridge: Cambridge University Press.

Van Valin, Robert D. \& Randy J. LaPolla. 1997. Syntax: Structure, Meaning and Function. Cambridge: Cambridge University Press.

Wulf, Alyssa, Paul Dudis, Robert Bailey \& Ceil Lucas. 2002. Variable subject presence in ASL narratives. Sign Language Studies 3(1), 54-76. 